\title{
Análisis de las necesidades de bienestar laboral de los empleados de universidades en la región caribe: Caso barranquilla
}

Analysis of the labor welfare needs of university employees in the caribbean region: the Barranquilla case

DOI: 10.21803/adgnosis.v8i8.361

Resumen

El bienestar laboral es un programa, conformado por actividades o estrategias, que redunde en beneficios para solucionar las necesidades reales del trabajador; dentro de la empresa, proyectado a la familia y a la comunidad donde se desempeña. Este articulo tiene por objetivo analizar las necesidades que presentan los empleados de las universidades de la región Caribe respecto al bienestar laboral. A través de un análisis de conglomerados jerárquicos se establecieron las fortalezas y amenazas que imperan en los programas de bienestar laboral de las universidades de la región Caribe dirigidas a sus empleados. Los resultados demuestran debilidades en un alto grado, en la esfera socio afectiva y familiar, en comparación a la esfera personal y ocupacional de bienestar laboral.

Palabras clave: Bienestar laboral, Región Caribe, Talento Humano

\section{Abstract}

Workplace well-being is a program, made up of activities or strategies, that results in benefits to solve the worker's real needs; within the company, projected to the family and the community where it works. The purpose of this article is to analyze the needs of the employees of the universities of the Caribbean region with regard to labor well-being. Through an analysis of hierarchical conglomerates, the strengths and threats that prevail in the labor welfare programs of the universities of the Caribbean region aimed at their employees were established. The results show weaknesses to a high degree, in the socioaffective and family sphere, in comparison to the personal and occupational sphere of labor well-being.

Keywords: Labor Welfare, Caribbean Region, Human Talent

\author{
Edwin Javier Arrieta-Valderrama ${ }^{1}$ \\ edwinarrietasena@gmail.com
}

Marcos Cabarcas Velasquez
mcabarcasv.at $@$ misena.edu.co

Carlos A. Rodríguez-Arias ${ }^{3}$

crodrigueza.91@gmail.com

\section{Cómo citar este artículo:}

Arrieta E., Cabarcas M. \& Rodríguez C. (2019). Análisis de las necesidades de bienestar laboral de los empleados de universidades en la región caribe: Caso barranquilla. Adgnosis, 8(8), p.73-90 DOI: 10.21803/adgnosis.v8i8.361

\footnotetext{
1 Administrador de Empresas de la Universidad Católica "Luis Amigó", Magister en Gerencia del Talento Humano de la Universidad Simón Bolivar, Instructor/Líder del Equipo ejecutor del Programa de Gestión Humana, del Centro de Comercio y Servicios, del Servicio Nacional de Aprendizaje SENA - Regional Atlántico. Profesor de la Universidad Americana - Sede Barranquilla, adscrito a la Facultad de Ciencias Económicas en el Programa Especialización en Gerencia Empresarial y Competitividad, se ha desempeñado como Facilitador de procesos en Gestión del Talento Humano en empresas privadas, pertenece al grupo de Investigación Arcadia - Minciencias, Coach Certificado y Facilitador Certificado en Herramientas de Aprendizaje Experiencial, Co-Creador en Colombia de la Herramienta de Coaching BIOPOLIS: E-mail: edwinarrietasena@gmail.com. Orcid: https://orcid.org/0000-0002-8936-0461

2 Administrador de Empresas de la Universidad del Atlántico, Magister en Administración de Empresas e Innovación de la Universidad, Especialista en Gerencia de Recursos humanos de la Universidad del Norte. Instructor, en el área de Gestión Humana, del Centro de Comercio y Servicios del Servicio Nacional de Aprendizaje SENA Regional Atlántico. Profesor de la Universidad del Atlántico en la Facultad de Ciencias Económicas en el programa de Administración de Empresas, en las áreas de administración, talento humano y jurado en trabajos de grado, se ha desempeñado como director del área de gestión de talento humano de varias empresas privadas de la ciudad de Barranquilla. Asesor de empresas. Líder de la línea de investigación "Innovación y Gestión Empresarial" del grupo de investigación Arcadia. E-mail: mcabarcasv.atl@misena.edu.co Orcid: https://orcid.org/0000-0001-9393-6067

3 Economista (Universidad de Cartagena), Magister en Ciencias de la Administración y las Organizaciones (Universidad Tecnológica de Bolívar). Investigador junior en Colciencias, adscrito al grupo Anfibios Innovadores de la Escuela de Formación de Infantería de Marina: E-mail: crodrigueza.91@gmail.com; Orcid: https://orcid.org/0000-0003-1567-9805
} 


\section{Introducción}

AA nivel mundial las organizaciones se han visto enfrentadas a un entorno cada vez más globalizado, donde las compañías han venido enfocando su interés en lograr sus objetivos, basados en el bienestar de sus colaboradores y en el cómo hacer sentir al trabajador parte fundamental de la empresa. De acuerdo a esto, se han venido formulando planes y programas para reivindicar, resaltar y darle la importancia que merece la labor que cumplen los empleados al interior de las organizaciones, de ahí que la gestión humana desde los departamento de bienestar al trabajador programen actividades y acciones relacionadas con la salud y seguridad en el trabajo, el desarrollo personal y familiar del empleado, elementos decisivos para afianzar la satisfacción, el sentido de pertenencia y el buen desempeño del empleado, sirviendo todo esto para la retención del talento humano en las compañías.

En este sentido, las universidades públicas y privadas en Colombia están viviendo un cambio en sus formas de operar y de administrarse, debido a la constante búsqueda de la calidad en sus procesos administrativos y académicos, que las lleven a las certificaciones nacionales e internacionales en buenas prácticas de procesos, y como parte fundamental se debe atender con mayor compromiso y dedicación al talento humano con que cuentan las instituciones de educación superior. Es así que, los programas de bienestar en las empresas se convierten en la piedra angular de la satisfacción laboral, pues los resultados de la falta de bienestar laboral pueden afectar la productividad de la organización y producir un deterioro en la calidad del entorno laboral. Puede disminuir el desempeño, incrementar el nivel de quejas, el ausentismo o el cambio de empleo (Estrada y Ramírez, 2010).

Para el caso de las universidades de la región Caribe a pesar que existen programas de bienestar laboral diseñados para mejorar la satisfacción laboral del empleado, en muchos casos no generan el impacto necesario acorde a su alcance. Con lo cual es importante verificar, desde la óptica del emplea- do, las fortalezas y debilidades de los programas de bienestar laboral empleados. Así las cosas, el objetivo del presente artículo es analizar las necesidades que presentan los empleados de las universidades de la región Caribe respecto al bienestar laboral con el fin de proponer estrategias que permitan estos programas.

\section{Revisión teórica}

El Bienestar laboral se considera como un proceso orientado a mejorar las condiciones de los empleados en las empresas, que favorecen a un desarrollo integral desde el ser, es decir, que impacta positivamente la dimensión interna y externa del trabajador. Desde la dimensión interna, los estímulos otorgados por los programas de bienestar laboral crean una satisfacción en el trabajo, haciendo cada vez más que se cree un sentido de pertenencia por parte del empleado, lo que conlleva a sentirse identificado con la organización, sobre todo en metas y objetivos organizacionales. Por otra parte, desde la dimensión externa, el bienestar laboral facilita la interacción del empleado con su entorno de trabajo, así mismo, con su núcleo familiar, permitiéndole un desarrollo social íntegro que se manifiesta en beneficios para la organización puesto que se aumenta la eficiencia, y eficiencia de los trabajadores mejorando el desempeño organizativo de cada uno.

En la actualidad la adecuada gestión del bienestar laboral en las empresas proporciona a los empleados mejoras en su calidad de vida laboral. Es por tanto que en esta investigación la relación entre estos dos conceptos se hace necesaria ya que se pretende abordar las dimensiones que comprende el bienestar laboral, así como sus indicadores.

Se iniciará por hacer un recorrido por el concepto teórico y evolutivo del bienestar laboral, se continuará con modelos referentes para la aplicación del Bienestar laboral, la importancia del programa con sus finalidades así mismo las dimensiones y la relación de este programa con la calidad de vida laboral. 


\subsection{Bienestar Laboral}

El bienestar laboral surge en respuesta a la preocupación de las condiciones del trabajo y de la baja satisfacción en el empleo por parte de las personas alrededor de los años 70 (Calderón, Murillo y Torres, 2003). Este se asocia comúnmente a la obtención de una remuneración (aparte del salario) por parte de los trabajadores por la prestación de sus servicios de trabajo. Sin embargo, este concepto va más allá de esta connotación económica y es que incluye al ser humano desde todas sus dimensiones; familiares, laborales, culturales, espirituales, etc. Estas, a pesar de que no están relacionadas directamente con aquellas habilidades (i.e., conocimiento, destreza) que tiene un trabajador para desarrollar una labor si influyen de manera indirecta en el desempeño individual de cada uno, lo que repercute en el desempeño para la organización.

Arenas (1993) afirma el bienestar laboral es un programa de servicios en beneficio al interior de las organizaciones, que satisfacen las necesidades de los trabajadores que fortalecen las relaciones internas y externas entre estos. De igual forma Vigoya (2002) define al bienestar laboral como el conjunto de programas que son estructurados para satisfacer necesidades de los trabajadores, ya que esta satisfacción influye en el reconocimiento por parte del individuo en pertenecer a un entorno social.

Al referirse a un Bienestar Laboral, se alude a un estado en donde los empleados gocen de salud física, mental, emocional y espiritual, para así obtener sus satisfacciones personales como familiares, aquí es donde el concepto de Bienestar Laboral y su importancia tanto para el empleado como para la empresa. Según Colciencias (2016) de acuerdo al programa de Bienestar 2016 de la Dirección de gestión de recursos y logística; manifiesta que el área de Calidad de Vida Laboral, se ocupara de fomentar un ambiente laboral satisfactorio y propicio para el Bienestar y el desarrollo del empleado; está constituida por las condiciones laborales relevantes para la satisfacción de las necesidades básicas del funcionamiento, la motivación y rendimiento laboral, logrando generar un impacto positivo al interior de la entidad, tanto en términos de productividad como en términos de relaciones interpersonales.

Con base en esto, y cómo ya se ha recalcado, el bienestar laboral comprende diversos aspectos de la vida del ser humano. Danna y Griffin (1999) consideran que el bienestar laboral comprende dos tipos de satisfacciones que disfrutan los individuos; la satisfacción en la vida, que comprende: la satisfacción y/o insatisfacción con la vida social, la vida familiar, la recreación, la espiritualidad, entre otras; y por otro lado la satisfacción en el trabajo que comprende la satisfacción y/o insatisfacción con el pago, oportunidades de ascenso, el trabajo en sí, compañeros de trabajo, entre otras). Además de este tipo de satisfacciones, hay otro componente importante que es la salud general del individuo, esta salud está determinada por indicadores psicológicos y fisiológicos.

En ese sentido, estos autores, afirman que del bienestar laboral preceden unas causas y son originarias también de unas consecuencias cómo se ve a continuación en la Figura 1:

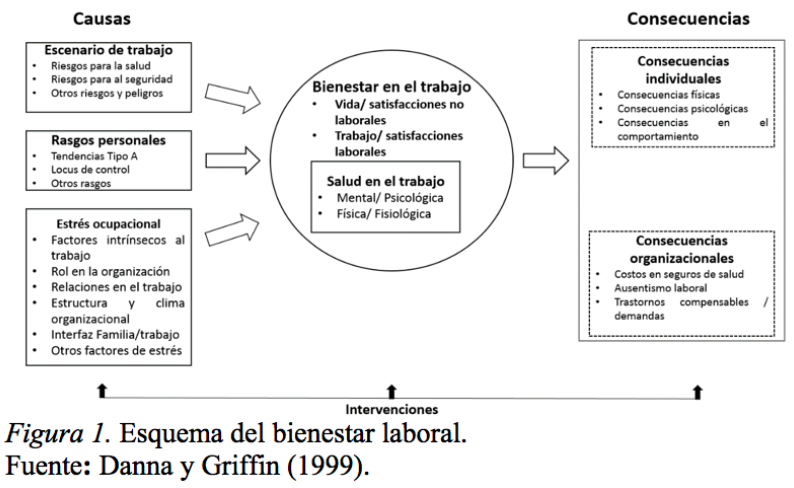

De acuerdo con la Figura 1 relacionada con el esquema del bienestar laboral, este se ve afectado por tres factores que anteceden su naturaleza. En primer lugar, el escenario de trabajo, el cual contiene riesgos para la salud, riesgos para la seguridad y otros riesgos y peligros, que pueden crear entornos de trabajo peligrosos, lo que, a su vez, tiene un impacto negativo en la salud y el bienestar de los tra- 
bajadores. Estos escenarios de trabajos en "riesgos" tienen una repercusión en los rasgos personales de manera positiva o negativa de los individuos.

Los rasgos de personalidad, sobre todo de tendencias de tipo $\mathrm{A}^{1}$, el locus de control y otras características, conjuntamente juegan un rol determinante en el grado de bienestar y salud laboral de un individuo en el entorno organizacional. Finalmente, dentro de las causas que pueden originar o no un bienestar laboral está el estrés laboral; dentro de estos factores de estrés se encuentran: Factores intrínsecos al trabajo, Rol en la organización, Relaciones con el trabajo, Estructura y Clima Organizacional, Interfaz Familia/Trabajo entre otros factores de estrés (Mejía Ceballos, Laca Arocena \& Gondra Rezola, 2009).

Dada estas condiciones entre las causas y consecuencias del bienestar laboral, la organización puede decidir dónde intervenir a fin de mejorar el bienestar laboral de sus empleados que se traduzcan en mejoras en la productividad, menos costes por el pago de seguros de salud y mayor desempeño organizacional. Las políticas en este caso pueden ser desde reactivas a activas. Las primeras consisten en intervenir las consecuencias que genera el bienestar laboral para mitigar los efectos negativos que pudieran tener los trabajadores.

Por su parte, las políticas activas se relacionan con la prevención de riesgos laborales que aumenten el bienestar en general de los trabajadores, por lo cual la intervención activa se constituye en una herramienta fundamental para que las empresas no solamente ofrezcan un bienestar laboral de calidad a sus trabajadores, sino que se constituye también en un factor que reduce los costos debido a que no se hará cargo de las consecuencias negativas que genere un ambiente laboral negativo tal como lo expresa (Martínez, 2008).

Es importante resaltar que el bienestar laboral ha presentado reformas y cambios laborales que han generado nuevos roles en las organizaciones, exigiéndoles a sus funcionarios eficiencia y eficacia en la realización de estos cambios ya que integrar los intereses de los trabajadores tanto para beneficios individuales cómo organizacionales no es tarea sencilla. A su vez los funcionarios que desempeñan una labor dentro de una organización, si no están necesariamente ligados afectivamente a la misma, estos podrían realizar con disgusto y malestar sus funciones y sentir desprecio hacia la organización. Situación que puede generar un bajo rendimiento, así como otras posibles consecuencias. Por esta razón, es de vital importancia que las organizaciones conozcan no solo el tipo, sino el grado de compromiso de sus miembros y una de las consecuencias es intentar elevarlo, teniendo en cuenta el aspecto que afecta el bienestar de sus funcionarios y su desarrollo de la vida profesional con las responsabilidades personales (Barrios y Paravic, 2006).

Todas estos esfuerzos apuntan a una satisfacción laboral del empleado; la cual está en función del apoyo social que le brinde la organización en la calidad de vida de cada empleado, incluyéndose en este proceso su núcleo familiar primario que deberá ser valorado, ya que estos actores pueden impactar positiva o negativamente en la autoestima, dado esto en el ambiente que se crean conductas y rutinas para mejorar la calidad de vida, disminuyendo los riesgos de salud física y psicológica, arrojando efectos de un mejor desarrollo personal, logrando una eficacia enfocada en el ámbito laboral y resultados estadísticos que benefician a la organización.

Resaltando este aspecto de familia y trabajo Frone, Russia y Cooper (1992) señalan la relación que existe entre el trabajo y la familia, siendo este tipo bidireccional, donde la influencia puede ser de conflicto o de facilitación. Estos autores, estudiaron la interface entre el trabajo y la familia, destacando que, en ocasiones, esa relación es de conflicto, pero en otros casos la relación establecida es de facilitación, ya que la familia ayuda al trabajador a enfrentar los problemas del trabajo, brindándole apoyo

1 Es un constructo teórico que se utiliza para denominar a un conjunto de respuestas conductuales, fisiológicas, cognitivas y emocionales que se manifiestan sobre todo en situaciones desafiantes. (García y Berrios, 1999, p. 358) 
emocional. Por tal razón, los programas de bienestar laboral en las organizaciones con frecuencia se extienden al núcleo familiar de sus trabajadores, pues en la medida que su entorno se sienta a gusto e involucrado con la organización, este sentido de pertenencia se afianza cada día más, para un mejor desarrollo en sus actividades laborales.

\section{Modelo para diseñar programas de bienestar laboral}

Con el propósito de crear un ámbito laboral ideal para el desarrollo y creación de procesos de formación para empleados del sector educativo con alto sentido de pertenencia y satisfacción por trabajar en un entorno sano que permita el crecimiento en todos los niveles, se plantea el siguiente modelo para el diseño de programas de bienestar laboral (Ministerio de Educación Nacional, 2009).

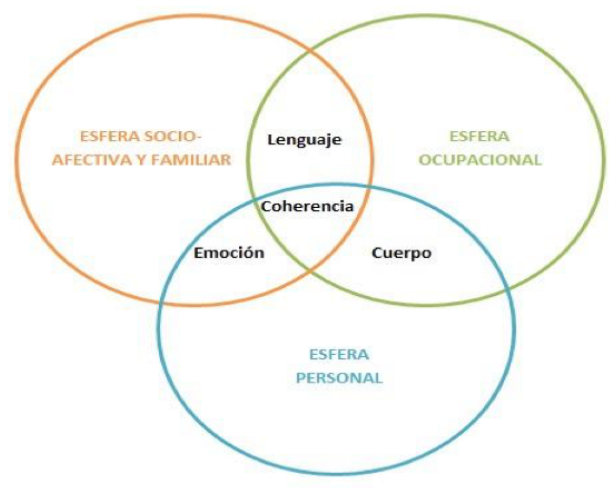

Figura 2. Modelo para el diseño de programas de bienestar laboral docente. Fuente: Ministerio de Educación Nacional (2009).

Este modelo de Coaching tiene como objetivo principal "Proporcionar a los líderes, una estrategia para movilizar y orientar el recurso humano, enfocando su inteligencia, su compromiso, su energía, y sus sentimientos hacia el logro de un objetivo común en beneficio de todos" (Ministerio de Educación Nacional, 2009, p. 7).

La intervención en cada una de las esferas representará calidad de vida, bienestar laboral y productividad, sus características, y algunas actividades sugeridas son descritas continuación.

\section{Esfera personal}

Abarca cada una de las acciones que tienen como objetivo la formación integral del trabajador como persona y que le permiten desenvolverse en su vida profesional.

Los agentes más importantes que se destacan en la esfera personal son:

\section{Desarrollo de carrera}

Son actividades que permiten identificar las áreas de proyección del trabajador con el fin de aplicar allí sus intereses y capacidades de logro y acción, de liderazgo y dirección, cognitivas, entre otras. Se sugiere el desarrollo de proyectos de vida y talleres para el fortalecimiento de las capacidades de los docentes.

\section{Desvinculación laboral asistida}

Consolidación de un programa de retiro asistido de los docentes y directivos de manera que la desvinculación sea lo menos traumática posible. Se sugieren programas que vinculen aspectos emocionales, ocupacionales, financieros, familiares y la preparación para un nuevo abordaje laboral.

\section{Educación formal}

Vinculación a la educación formal por medio del establecimiento de convenios con entidades educativas, inclusión de becas de educación formal para empleados y establecimiento de convenios con entidades crediticias (Ministerio de Educación Nacional, 2009).

\section{Esfera socio-afectiva y familiar}

Permite el mejoramiento de la calidad de vida por medio de garantías en las condiciones de seguridad física, emocional y social por medio del desarrollo de habilidades y destrezas.

Los agentes más importantes que se destacan en la esfera socio afectivo son:

\section{Seguridad social integral}

Es el conjunto de instituciones, normas y procedimientos con los que cuenta el personal para gozar 
de calidad de vida en el entorno laboral. Se sugieren talleres de promoción de la salud, jornadas de exámenes médicos, talleres de régimen pensional, riesgos profesionales y jornadas de asesoría en temas de salud, servicios de caja de compensación y vivienda.

\section{Recreación y cultura}

Generación de ambientes que ayuden al reconocimiento de capacidades de expresión, imaginación, comunicación y desarrollo. Se sugiere la promoción de actividades culturales como la danza, música, canto, teatro, poesía, pintura, actividades deportivas, ecológicas, programas de acondicionamiento físico, vacaciones recreativas, entre otras. (Ministerio de Educación Nacional, 2009).

\section{Esfera ocupacional}

Garantiza que el trabajador pueda desempeñar su labor bajo las mejores condiciones físicas, ambientales y emocionales para afianzar su compromiso e identidad para con la institución (Ministerio de Educación Nacional, 2009).

Los agentes más importantes que se destacan en la esfera socio ocupacional son:

\section{Clima organizacional}

Se toman en cuenta las experiencias personales de cada uno de los trabajadores, sus necesidades, motivaciones, deseos, expectativas y la implementación de programas que estimulen el clima laboral. Se sugiere medir el clima organizacional máximo cada dos años, realizar sondeos de intereses, estudios de diagnóstico de servicio y el estudio de perfiles de los puestos de trabajo.

\section{Incentivos}

Se deberá premiar la excelencia, aquellos empleados cuyo desempeño sea evaluado como superior deberán recibir incentivos, a su vez, el desempeño deberá ir encaminado a la consecución de la mejora de métodos de enseñanza, en el caso del sector educativo, y la creación de conciencia de responsabilidad social docente. Se sugiere diseñar un plan de incentivos territorial, reconocimiento público a los mejores por elección del estudiantado, reconocimiento por antigüedad y participación destacada en actividades extracurriculares.

\section{Estilos de dirección}

Se deberá revisar las formas de dirección que ejercen los superiores de manera que sea permitida la participación de los empleados y se aporte positivamente a la calidad de vida con los ajustes requeridos. Se sugieren talleres para mejorar el desarrollo de habilidades gerenciales como liderazgo, comunicación, relaciones interpersonales y toma de decisiones, a su vez aumentar la frecuencia de reuniones para tratar temas laborales, contribuyendo así a la comunicación y el trabajo en equipo.

\section{Cultura organizacional}

Socialización del funcionamiento al interior de la organización con los servidores para aumentar la autocracia, delegación y control. Se sugiere realizar estudios de medición de la cultura organizacional con periodicidad menor a dos (2) años, garantizar la participación de los empleados en programas de inducción y reinducción, establecimiento de carta de valores institucionales, creación de himnos, lemas, e historias.

\section{Gerencia del cambio}

Proceso de reflexión y decisiones personales para asumir cambios en las condiciones internas y externas en el ámbito laboral y personal. Se sugiere diseñar una estrategia que resulte de un proceso de acciones concertadas por los participantes del proceso, que sea capaz de movilizar recursos preexistentes en las personas y entidades aún no desarrollados, que conlleven cambios que repercutan positivamente al entorno en general y que integre dimensiones físicas, emocionales, cognoscitivas, espirituales y operativas.

\section{Solución de conflictos}

Es necesario que el conflicto sea llevado de la mejor manera para estimular a los individuos a buscar métodos de solución y mantener el buen funcionamiento del entorno laboral. Se sugiere realizar programas preventivos en manejo de riesgos psico- 
sociales, formativos en estilos de dirección, comités de convivencia bipartito que actúen en situaciones de conflicto, programación neurolingüística y acompañamiento a agresores y agredidos.

\section{Salud ocupacional}

Tienen como objetivo mantener la salud física, mental y social de los servidores en los puestos de trabajo proporcionando condiciones seguras e higiénicas. Estos programas deben llevarse a cabo en coordinación con la administradora de riesgos profesionales (ARP) vinculada a la entidad. Se sugiere conformar un comité paritario de salud ocupacional, medir los factores de riesgo psicosocial, estudios de perfil demográfico y morbilidad sentida y un programa de pausa laboral activa (Ministerio de Educación Nacional, 2009).

\section{Diseño metodológico}

Esta investigación se enmarca en lo que la Fundación Española de Ciencia y Tecnología (FECYT, 2002) investigación aplicada, en este tipo de investigación se presentan principalmente trabajos originales que se enfocan en un objetivo práctico específico.

La variable de estudio es el Bienestar Laboral, esta se medirá de acuerdo a unas sub variables e indicadores con base en el modelo del Ministerio de Educación Nacional (2009). El cual determina que el bienestar laboral está compuesto de tres subvariables: esfera socio afectiva, familiar, Esfera Personal y Esfera Ocupacional.

Para el primer caso, los indicadores de medición de esta subvariable son: programas de promoción y prevención, ocio y cultura, apoyos asistenciales y Accesos a Créditos. Así mismo se tuvo en cuenta variables de Control cómo: Edad, Género, Nivel Educativo, Cargo, Área, Años de Antigüedad, Horas de trabajo diario, Estado civil e Hijos.

Las variables son de tipo ordinal principalmente puesto que se utilizarán escalas Likert en la medición de los indicadores. Esta escala irá entre los valores de uno a cinco. Por otra parte, las variables de control serán de tipo intervalo, ordinal y nominal respectivamente.

Se seleccionó una universidad para desarrollar la investigación la cual se relaciona a continuación en la siguiente tabla 1:

Tabla 1.

Descripción del personal administrativo

\begin{tabular}{cc}
\hline \multicolumn{2}{c}{ Personal Administrativo } \\
\hline Nivel & Número de personas \\
Operativo & 302 \\
Ejecutivo & 81 \\
Directivo & 8 \\
\hline
\end{tabular}

Fuente: Elaboración propia.

Debido a las características de la población, para determinar la muestra se hace necesario aplicar la técnica de muestreo probabilístico estratificado ya que aumentaría la precisión de la muestra seleccionado en sus diferentes categorías (Hernández, Fernández y Baptista, 2010) es decir, para este caso sería determinar las muestras para cada nivel (Operativo, ejecutivo y Directivo).

Por otra parte, para la identificación de la ecuación muestral, se realizó mediante muestras para poblaciones finitas (Hernández, Fernández y Baptista, 2010).

En este sentido, las muestras vendrían determinadas así:

\section{Cálculo de la Muestra para el Nivel Operativo}

ecuación (1) $\frac{\mathrm{N} * \mathrm{Z}^{2} * \mathrm{p} * \mathrm{q}}{\mathrm{d}^{2} *(\mathrm{~N}-1)^{2}+\mathrm{Z}^{2} * \mathrm{p} * \mathrm{q}}=\frac{302 * 1,96^{2} * 0.5 * 0.5}{0.05^{2}(302-1)+1,96^{2} * 0.5 * 0.5}=169$

De acuerdo a la ecuación (1); Con un error del $5 \%$ y un nivel de confianza del $95 \%$, con una seguridad $\mathrm{Z}$ del $95 \%$ se tendrá un valor de 1.96, con una distribución probabilidades de un $50 \%$ para cada evento, la muestra resultante será de 169 trabajadores administrativos en el nivel operativo de la Universidad. 


\section{Cálculo de la Muestra para el Nivel Ejecutivo}

ecuación (2) $\frac{\mathrm{N} * \mathrm{Z}^{2} * \mathrm{p} * \mathrm{q}}{\mathrm{d}^{2} *(\mathrm{~N}-1)^{2}+\mathrm{Z}^{2} * \mathrm{p} * \mathrm{q}}=\frac{81 * 1,96^{2} * 0.5 * 0.5}{0.05^{2}(81-1)+1,96^{2} * 0.5 * 0.5}=67$

De acuerdo a la ecuación (2); Con un error del $5 \%$ y un nivel de confianza del $95 \%$, con una seguridad $Z$ del $95 \%$ se tendrá un valor de 1.96, con una distribución probabilidades de un $50 \%$ para cada evento, la muestra resultante será de 67 trabajadores administrativos en el nivel ejecutivo de la Universidad.

\section{Cálculo de la Muestra para el Nivel Directivo}

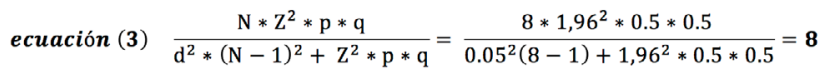

De acuerdo a la ecuación (3); Con un error del 5\% y un nivel de confianza del 95\%, con una seguridad $Z$ del $95 \%$ se tendrá un valor de 1.96, con una distribución probabilidades de un $50 \%$ para cada evento, la muestra resultante será de 8 trabajadores administrativos en el Nivel Directivo de la Universidad

Para la obtención de la información primaria necesaria para el estudio se aplicará mediante encuestas, se tendrá como referencia la encuesta propuesta por Meliá y Peiró (1989) donde especifican componentes a tener en cuenta desde las dimensiones: socio afectiva familiar; Personal; y Ocupacional. Se hará necesario aplicar una prueba piloto para luego realizar un test de validez y confiabilidad del instrumento usando el estadístico alfa de Cronbach.

En cuanto a la información secundaria, se apoyará la investigación con información proveniente de la Universidad, específicamente del programa de bienestar laboral. Así mismo, con libros, artículos científicos y tesis encontradas en los recursos electrónicos de la Universidad. Finalmente, se tendrán en cuenta documentos fundantes en el área de bienestar laboral proporcionados por las bases de datos de la Web of Science SCOPUS principalmente, ya que aquí es donde se genera mayormente el conocimiento científico.
Para la recolección de la información que permita responder a los objetivos propuestos se plantea un instrumento tipo de encuesta, el cual está dividido en dos partes; la primera parte unas preguntas sociodemográficas que permitirán obtener una caracterización del encuestado: la segunda parte está compuesta por preguntas tipo escala Likert. La escala que se plantea, se presenta desde valoraciones de uno a cinco: siendo uno $=$ nunca; dos $=$ casi nunca; tres $=$ mu$^{-}$ chas veces; cuatro $=$ casi siempre y cinco $=$ Siempre . Esta segunda parte se divide desde las tres esferas del bienestar laboral mencionadas anteriormente: Esfera socio afectiva y familiar; Esfera Personal y Esfera Ocupacional.

De otra parte, para la validación del instrumento a aplicar se realizó el test de fiabilidad y consistencia interna de instrumento, Alfa de Cronbach. Este plantea unas condiciones: si el estadístico Alfa de Cronbach $<0,69$ no existe una buena consistencia interna de los ítems (i.e. las preguntas de la encuesta) por lo tanto estas no miden lo que se requiere medir. Por otro lado, si el Alfa de Cronbach se encuentra entre los intervalos 0,7-0,9 se considera aceptable y el instrumento presenta una buena consistencia interna (Campo-Arias y Oviedo, 2008).

En este sentido, los resultados del Alfa de Cronbach se presentan en la Tabla 2 :

Tabla 2.

Análisis de fiabilidad

\begin{tabular}{|c|c|c|}
\hline \multicolumn{3}{|c|}{ Estadísticas de fiabilidad } \\
\hline & Alfa de Cronbach & \\
\hline & basada en & \\
\hline Alfa de & elementos & $\mathrm{N}$ de \\
\hline Cronbach & estandarizados & elementos \\
\hline, 803 & ,873 & 33 \\
\hline
\end{tabular}

Fuente: Elaboración propia.

Se puede inferir que de acuerdo a los 33 ítems de la encuesta, la prueba piloto arrojó un valor de 0.803 en el estadístico Alfa de Cronbach, lo que indica una buena confiabilidad, dicho en otras palabras, una buena consistencia interna del instrumento ya que 
éste está en el rango entre 0,7-0,9. Dado el valor obtenido se afirma que el instrumento nos permite discriminar entre los sujetos y que responde a las necesidades de lo que se pretende medir.

El método de análisis corresponderá inicialmente a las frecuencias de los ítems que conforman cada una de las variables, se utiliza la siguiente convención de colores Rojo para indicar las concentraciones bajas de frecuencias, es decir donde se evidencian las opiniones de la menor cantidad de sujetos, y con color verde que refleja una mayor cantidad de sujetos.

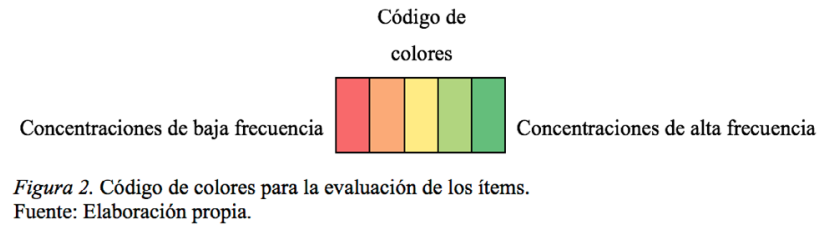

Por otra parte, con el fin de reagrupar los ítems en términos de fortalezas y debilidades se utiliza la técnica de conglomerados jerárquicos conformándose dos conglomerados. Se presenta el criterio para definir la inclusión de un ítem en los conglomerados:

\section{Respuestas Negativas $=\sum$ (Sumatoria): res- puestas "Nunca" y "Casi nunca" por parte de los encuestados.}

\section{Respuestas Positivas $=\sum$ (Sumatoria): res- puestas "casi siempre" y "Siempre" por parte de los encuestados.}

\section{Respuestas neutras $=\sum$ (Sumatoria): res- puestas “neutras" por parte de los encues- tados.}

Figura 3. ítems de los conglomerados.

Fuente: Elaboración propia.

La condición para declarar un ítem como debilidad, neutralidad o fortaleza se realiza así:

1. Si las respuestas negativas por parte de los encuestados son mayores que las respuestas positi- vas y las respuestas neutras, entonces existe condición de debilidad del ítem.

2. Si las respuestas positivas son mayores que las respuestas negativas y las respuestas neutras, entonces existe condición de fortaleza del ítem.

3. Si las respuestas neutras son mayores que las respuestas negativas y las respuestas positivas entonces, existe condición de neutralidad en el ítem.

\section{Resultados}

La primera esfera analizada es la Socio afectiva y familiar, conformada por 14 ítems. En ella se evidencian que en los ítems donde la mayoría de los encuestados manifiesta que los sucesos que se indagan en dichos ítems se presentan con frecuencia son: Muestra interés por las capacitaciones sobre autocuidado, higiene personal, autoestima y relaciones interpersonales programadas por Bienestar Laboral, $73 \%$ y Considera que las actividades programadas por Bienestar Laboral fortalecen la relación trabajo-familia 79,2\%, para el resto de variables la mayor concentración de sujetos se centra en que ocurren con poca frecuencia como se evidencia en la tabla 7 la cual muestra las frecuencias y porcentajes de los ítems.

La segunda esfera analizada es la esfera personal conformada por 7 ítems mostrándose que los que revisten debilidad de acuerdo al código de colores son: Ha recibido apoyo del "Centro de Servicios Externos" perteneciente a la Universidad para el desarrollo de su proyecto de vida, Ha accedido a los beneficios de formación académica establecidos por la Universidad, ha utilizado los medios que le brinda la Universidad para acceder a entidades externas, para préstamos por libranza, vivienda, y/o convenios empresariales, Ha recibido capacitaciones y orientaciones por parte de la Universidad, que lo prepare a la etapa de jubilación.

A continuación, se muestra la tabla 4 que muestra la distribución de las frecuencias de la esfera personal. 
Tabla 3.

Frecuencias absolutas y porcentuales de los items que conforman la esfera socio afectiva y familiar

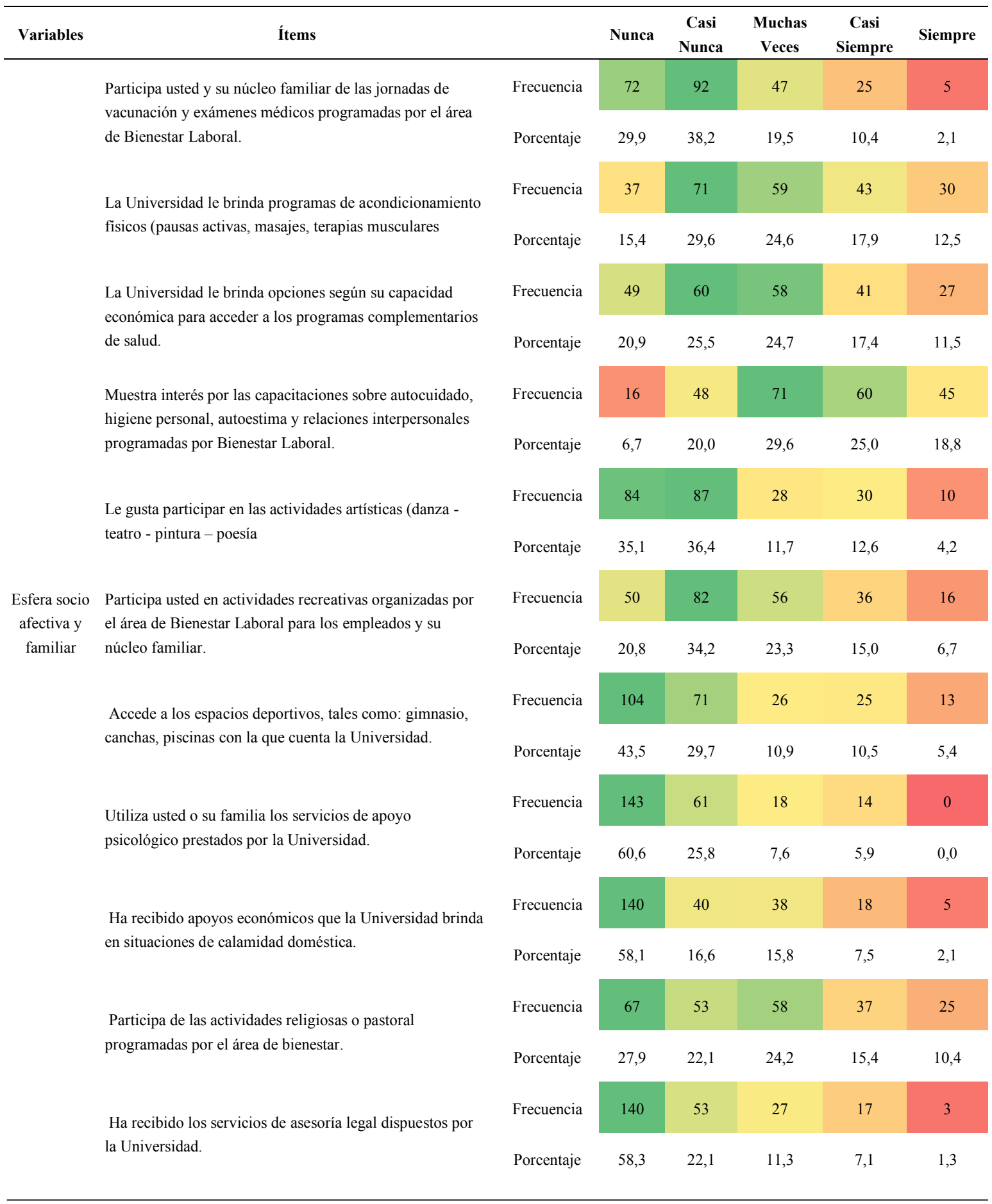




\begin{tabular}{|c|c|c|c|c|c|c|c|}
\hline Variables & Ítems & & Nunca & $\begin{array}{c}\text { Casi } \\
\text { Nunca }\end{array}$ & $\begin{array}{c}\text { Muchas } \\
\text { Veces }\end{array}$ & $\begin{array}{c}\text { Casi } \\
\text { Siempre }\end{array}$ & Siempre \\
\hline \multirow{2}{*}{\multicolumn{2}{|c|}{$\begin{array}{l}\text { Su núcleo familiar ha tenido la oportunidad de emplearse } \\
\text { laboralmente al interior de la Universidad. }\end{array}$}} & Frecuencia & 152 & 47 & 20 & 16 & 6 \\
\hline & & Porcentaje & 63,1 & 19,5 & 8,3 & 6,6 & 2,5 \\
\hline \multirow{2}{*}{\multicolumn{2}{|c|}{$\begin{array}{l}\text { Su núcleo familiar ha recibido apoyos educativos por parte } \\
\text { de la Universidad. }\end{array}$}} & Frecuencia & 119 & 41 & 44 & 21 & 14 \\
\hline & & Porcentaje & 49,8 & 17,2 & 18,4 & 8,8 & 5,9 \\
\hline \multirow{2}{*}{\multicolumn{2}{|c|}{$\begin{array}{l}\text { Considera que las actividades programadas por Bienestar } \\
\text { Laboral fortalecen la relación trabajo-familia. }\end{array}$}} & Frecuencia & 9 & 41 & 63 & 58 & 69 \\
\hline & & Porcentaje & 3,8 & 17,1 & 26,3 & 24,2 & 28,8 \\
\hline
\end{tabular}

Fuente: Elaboración propia.

Tabla 4.

Frecuencias absolutas y relativas de los items que conforman la esfera personal

\begin{tabular}{|c|c|c|c|c|c|c|c|}
\hline Variables & Ítems & & Nunca & $\begin{array}{c}\text { Casi } \\
\text { Nunca }\end{array}$ & $\begin{array}{c}\text { Muchas } \\
\text { Veces }\end{array}$ & $\begin{array}{c}\text { Casi } \\
\text { Siempre }\end{array}$ & Siempre \\
\hline \multirow{14}{*}{$\begin{array}{c}\text { Esfera } \\
\text { personal }\end{array}$} & $\begin{array}{l}\text { Ha recibido apoyo del "Centro de } \\
\text { Servicios Externos" perteneciente a la }\end{array}$ & Frecuencia & 100 & 74 & 39 & 15 & 11 \\
\hline & $\begin{array}{l}\text { Universidad para el desarrollo de su } \\
\text { proyecto de vida. }\end{array}$ & Porcentaje & 41,8 & 31,0 & 16,3 & 6,3 & 4,6 \\
\hline & $\begin{array}{l}\text { Ha accedido a los beneficios de } \\
\text { formación académica establecidos }\end{array}$ & Frecuencia & 63 & 55 & 59 & 29 & 33 \\
\hline & por la Universidad. & Porcentaje & 26,4 & 23,0 & 24,7 & 12,1 & 13,8 \\
\hline & $\begin{array}{c}\text { Ha utilizado los medios que le brinda } \\
\text { la Universidad para acceder a }\end{array}$ & Frecuencia & 98 & 54 & 31 & 20 & 36 \\
\hline & $\begin{array}{l}\text { entidades externas, para préstamos } \\
\text { por libranza, vivienda, y/o convenios } \\
\text { empresariales. }\end{array}$ & Porcentaje & 41,0 & 22,6 & 13,0 & 8,4 & 15,1 \\
\hline & $\begin{array}{l}\text { Ha participado en algún proceso de } \\
\text { formación o actualización en }\end{array}$ & Frecuencia & 39 & 39 & 78 & 50 & 31 \\
\hline & competencias laborales. & Porcentaje & 16,5 & 16,5 & 32,9 & 21,1 & 13,1 \\
\hline & $\begin{array}{l}\text { Recibe reconocimiento por su } \\
\text { desempeño por parte de la }\end{array}$ & Frecuencia & 45 & 42 & 48 & 70 & 33 \\
\hline & Universidad. & Porcentaje & 18,9 & 17,6 & 20,2 & 29,4 & 13,9 \\
\hline & $\begin{array}{l}\text { Ha recibido capacitaciones y } \\
\text { orientaciones por parte de la }\end{array}$ & Frecuencia & 103 & 47 & 27 & 30 & 33 \\
\hline & $\begin{array}{l}\text { Universidad que lo prepare a la etapa } \\
\text { de jubilación. }\end{array}$ & Porcentaje & 42,9 & 19,6 & 11,3 & 12,5 & 13,8 \\
\hline & $\begin{array}{c}\text { El programa de Bienestar Laboral } \\
\text { organiza actividades que desarrollan }\end{array}$ & Frecuencia & 24 & 51 & 70 & 37 & 58 \\
\hline & $\begin{array}{c}\text { sus habilidades y destrezas para un } \\
\text { mejor desempeño profesional y } \\
\text { laboral. }\end{array}$ & Porcentaje & 10,0 & 21,3 & 29,2 & 15,4 & 24,2 \\
\hline
\end{tabular}

Fuente: Elaboración propia 
Finalmente se muestra el análisis de las frecuencias de la esfera ocupacional, ésta es la esfera que reviste mayores fortalezas, el ítem que muestra menos fortaleza de acuerdo a la convención de colores utilizadas es: Se ha beneficiado de las políticas de promoción y ascensos instituidos por la Universidad.

Tabla 5 .

Frecuencias absolutas y porcentuales de la esfera ocupacional

\begin{tabular}{|c|c|c|c|c|c|c|c|}
\hline Variables & Ítems & & Nunca & $\begin{array}{c}\text { Casi } \\
\text { Nunca }\end{array}$ & $\begin{array}{c}\text { Muchas } \\
\text { Veces }\end{array}$ & $\begin{array}{c}\text { Casi } \\
\text { Siempre }\end{array}$ & Siempre \\
\hline \multirow{24}{*}{$\begin{array}{c}\text { Esfera } \\
\text { ocupaciona } \\
1\end{array}$} & \multirow{2}{*}{$\begin{array}{c}\text { El salario que devenga está acorde a las } \\
\text { funciones que realiza dentro de la } \\
\text { Universidad. }\end{array}$} & Frecuencia & 24 & 46 & 59 & 72 & 38 \\
\hline & & Porcentaje & 10,0 & 19,2 & 24,7 & 30,1 & 15,9 \\
\hline & \multirow{2}{*}{$\begin{array}{l}\text { Considera que la Universidad le } \\
\text { proporciona estabilidad laboral. }\end{array}$} & Frecuencia & 4 & 10 & 40 & 79 & 107 \\
\hline & & Porcentaje & 1,7 & 4,2 & 16,7 & 32,9 & 44,6 \\
\hline & \multirow{2}{*}{$\begin{array}{l}\text { La Universidad le ofrece óptimas } \\
\text { condiciones físicas de trabajo. }\end{array}$} & Frecuencia & 3 & 13 & 51 & 80 & 92 \\
\hline & & Porcentaje & 1,3 & 5,4 & 21,3 & 33,5 & 38,5 \\
\hline & \multirow{2}{*}{$\begin{array}{l}\text { Encuentra en la Universidad un buen } \\
\text { clima laboral. }\end{array}$} & Frecuencia & 2 & 1 & 50 & 76 & 111 \\
\hline & & Porcentaje & 0,8 & 0,4 & 20,8 & 31,7 & 46,3 \\
\hline & \multirow{2}{*}{$\begin{array}{l}\text { Se siente motivado con los incentivos } \\
\text { brindados por la Universidad. }\end{array}$} & Frecuencia & 17 & 27 & 70 & 78 & 49 \\
\hline & & Porcentaje & 7,1 & 11,2 & 29,0 & 32,4 & 20,3 \\
\hline & \multirow{2}{*}{$\begin{array}{l}\text { El estilo de dirección en la Universidad } \\
\text { se caracteriza por ser de puertas abiertas. }\end{array}$} & Frecuencia & 6 & 17 & 60 & 83 & 74 \\
\hline & & Porcentaje & 2,5 & 7,1 & 25,0 & 34,6 & 30,8 \\
\hline & \multirow{2}{*}{$\begin{array}{l}\text { Cuenta con las herramientas necesarias } \\
\text { de seguridad y salud en el trabajo para el } \\
\text { cumplimiento de sus funciones. }\end{array}$} & Frecuencia & 4 & 11 & 52 & 80 & 92 \\
\hline & & Porcentaje & 1,7 & 4,6 & 21,8 & 33,5 & 38,5 \\
\hline & \multirow{2}{*}{$\begin{array}{l}\text { Participa usted en capacitaciones } \\
\text { relacionadas con el programa de } \\
\text { seguridad y salud en el trabajo. }\end{array}$} & Frecuencia & 33 & 53 & 44 & 54 & 57 \\
\hline & & Porcentaje & 13,7 & 22,0 & 18,3 & 22,4 & 23,7 \\
\hline & \multirow{2}{*}{$\begin{array}{l}\text { La Universidad le cumple con los } \\
\text { requisitos legales en seguridad social } \\
\text { (ARL, SALUD Y PENSION }\end{array}$} & Frecuencia & 3 & 13 & 24 & 30 & 171 \\
\hline & & Porcentaje & 1,2 & 5,4 & 10,0 & 12,4 & 71,0 \\
\hline & \multirow{2}{*}{$\begin{array}{l}\text { Se ha beneficiado de las políticas de } \\
\text { promoción y ascensos instituidos por la } \\
\text { Universidad. }\end{array}$} & Frecuencia & 77 & 53 & 38 & 43 & 28 \\
\hline & & Porcentaje & 32,2 & 22,2 & 15,9 & 18,0 & 11,7 \\
\hline & \multirow{2}{*}{$\begin{array}{c}\text { La Universidad comunica a través de } \\
\text { los diferentes medios, las actividades } \\
\text { programadas por el área de Bienestar } \\
\text { Laboral. }\end{array}$} & Frecuencia & 6 & 20 & 77 & 49 & 88 \\
\hline & & Porcentaje & 2,5 & 8,3 & 32,1 & 20,4 & 36,7 \\
\hline & \multirow{2}{*}{$\begin{array}{l}\text { Las actividades desarrolladas por el } \\
\text { programa de Bienestar Laboral, llenan } \\
\text { sus expectativas y la de su familia. }\end{array}$} & Frecuencia & 6 & 35 & 71 & 68 & 61 \\
\hline & & Porcentaje & 2,5 & 14,5 & 29,5 & 28,2 & 25,3 \\
\hline
\end{tabular}

Fuente: Elaboración propia. 
Un primer conglomerado está compuesto por las variables que no se evidencian fortalezas y en las cuales se hace necesaria realizar una intervención para mejorar los tópicos que se aborda en cada uno de ellos. A continuación, se muestra el primer conglomerado en la tabla 6

Tabla 6.

Primer conglomerado - Debilidades

\begin{tabular}{|c|c|c|c|c|c|c|c|}
\hline No. & ITEMS & $\begin{array}{l}\text { Respuestas } \\
\text { Negativas }\end{array}$ & $\begin{array}{c}\text { Respuestas } \\
\text { Neutras }\end{array}$ & $\begin{array}{l}\text { Respuestas } \\
\text { Positivas }\end{array}$ & $\begin{array}{c}\text { Condición } \\
\text { de Debilidad }\end{array}$ & $\begin{array}{c}\text { Conglom } \\
\text { erado }\end{array}$ & $\begin{array}{l}\text { Esfera } \\
\text { Inicial }\end{array}$ \\
\hline 1 & $\begin{array}{l}\text { Participa usted y su núcleo familiar de } \\
\text { las jornadas de vacunación y exámenes } \\
\text { médicos programadas por el área de } \\
\text { Bienestar Laboral. }\end{array}$ & 164 & 47 & 30 & SI & 1 & $\begin{array}{c}\text { Esfera } \\
\text { socioafectiva } \\
\text { y familiar }\end{array}$ \\
\hline 2 & $\begin{array}{l}\text { La Universidad le brinda programas de } \\
\text { acondicionamiento físicos (pausas } \\
\text { activas, masajes, terapias musculares }\end{array}$ & 108 & 59 & 73 & SI & 1 & $\begin{array}{c}\text { Esfera } \\
\text { socioafectiva } \\
\text { y familiar }\end{array}$ \\
\hline 3 & $\begin{array}{l}\text { La Universidad le brinda opciones } \\
\text { según su capacidad económica para } \\
\text { acceder a los programas } \\
\text { complementarios de salud. }\end{array}$ & 109 & 58 & 68 & SI & 1 & $\begin{array}{c}\text { Esfera } \\
\text { socioafectiva } \\
\text { y familiar }\end{array}$ \\
\hline 5 & $\begin{array}{l}\text { Le gusta participar en las actividades } \\
\text { artísticas (danza - teatro - pintura - } \\
\text { poesía }\end{array}$ & 171 & 28 & 40 & SI & 1 & $\begin{array}{c}\text { Esfera } \\
\text { socioafectiva } \\
\text { y familiar }\end{array}$ \\
\hline 6 & $\begin{array}{l}\text { Participa usted en actividades } \\
\text { recreativas organizadas por el área de } \\
\text { Bienestar Laboral para los empleados } \\
\text { y su núcleo familiar. }\end{array}$ & 132 & 56 & 52 & SI & 1 & $\begin{array}{c}\text { Esfera } \\
\text { socioafectiva } \\
\text { y familiar }\end{array}$ \\
\hline 7 & $\begin{array}{l}\text { Accede a los espacios deportivos, } \\
\text { tales como: gimnasio, canchas, } \\
\text { piscinas con la que cuenta la } \\
\text { Universidad. }\end{array}$ & 175 & 26 & 38 & SI & 1 & $\begin{array}{c}\text { Esfera } \\
\text { socioafectiva } \\
\text { y familiar }\end{array}$ \\
\hline 8 & $\begin{array}{l}\text { Utiliza usted o su familia los servicios } \\
\text { de apoyo psicológico prestados por la } \\
\text { Universidad. }\end{array}$ & 204 & 18 & 14 & SI & 1 & $\begin{array}{c}\text { Esfera } \\
\text { socioafectiva } \\
\text { y familiar }\end{array}$ \\
\hline 9 & $\begin{array}{l}\text { Ha recibido apoyos económicos que la } \\
\text { Universidad brinda en situaciones de } \\
\text { calamidad doméstica. }\end{array}$ & 180 & 38 & 23 & SI & 1 & $\begin{array}{c}\text { Esfera } \\
\text { socioafectiva } \\
\text { y familiar }\end{array}$ \\
\hline 10 & $\begin{array}{l}\text { Participa de las actividades religiosas } \\
\text { o pastoral programadas por el área de } \\
\text { bienestar. }\end{array}$ & 120 & 58 & 62 & SI & 1 & $\begin{array}{c}\text { Esfera } \\
\text { socioafectiva } \\
\text { y familiar }\end{array}$ \\
\hline 11 & $\begin{array}{l}\text { Ha recibido los servicios de asesoría } \\
\text { legal dispuestos por la Universidad. }\end{array}$ & 193 & 27 & 20 & SI & 1 & $\begin{array}{c}\text { Esfera } \\
\text { socioafectiva } \\
\text { y familiar }\end{array}$ \\
\hline 12 & $\begin{array}{l}\text { Su núcleo familiar ha tenido la } \\
\text { oportunidad de emplearse } \\
\text { laboralmente al interior de la } \\
\text { Universidad. }\end{array}$ & 199 & 20 & 22 & SI & 1 & $\begin{array}{l}\text { Esfera } \\
\text { socioafectiva } \\
\text { y familiar }\end{array}$ \\
\hline 13 & $\begin{array}{l}\text { Su núcleo familiar ha recibido apoyos } \\
\text { educativos por parte de la Universidad. }\end{array}$ & 160 & 44 & 35 & SI & 1 & $\begin{array}{c}\text { Esfera } \\
\text { socioafectiva } \\
\text { y familiar }\end{array}$ \\
\hline 15 & $\begin{array}{l}\text { Ha recibido apoyo del "Centro de } \\
\text { Servicios Externos" perteneciente a la } \\
\text { Universidad para el desarrollo de su } \\
\text { proyecto de vida. }\end{array}$ & 174 & 39 & 26 & SI & 1 & $\begin{array}{c}\text { Esfera } \\
\text { personal }\end{array}$ \\
\hline
\end{tabular}




$\mathbf{1 6} \begin{aligned} & \text { Ha accedido a los beneficios de } \\ & \text { formación académica establecidos por } \\ & \text { la Universidad. } \\ & \text { Ha utilizado los medios que le brinda } \\ & \text { la Universidad para acceder a } \\ & \text { entidades externas, para préstamos por } \\ & \text { libranza, vivienda, y/o convenios } \\ & \text { empresariales. }\end{aligned}$
$\begin{aligned} & \text { Ha recibido capacitaciones y } \\ & \text { orientaciones por parte de la } \\ & \text { Universidad que lo prepare a la etapa } \\ & \text { de jubilación. }\end{aligned}$
$\begin{aligned} & \text { Se ha beneficiado de las políticas de } \\ & \text { promoción y ascensos instituidos por } \\ & \text { la Universidad. }\end{aligned}$

Fuente: elaboración propia.

Se puede observar de la tabla 6 que se encuentra solo un ítem relacionado con la esfera ocupacional. Así mismo la mayoría de las debilidades quedan resaltadas desde la esfera socio afectivo y familiar coincidiendo un total del $85,7 \%$ de los ítems evaluados en esta dimensión. Una explicación de estos resultados puede ser que, aunque el departamento hace uso de los medios de comunicación para dar a conocer las diferentes actividades, observamos que el nivel de participación no genera el impacto deseado, lo que puede indicar que los espacios o jornadas en los que se está realizando no son los adecuados o en su defecto no están claros algunos protocolos para acceder a los servicios. Así mismo, se evidencia desconocimiento de algunos beneficios con los que cuentan los empleados y tal vez estos no son divulgados eficientemente en los procesos de inducción o los mecanismos para acceder a los beneficios no están plenamente identificados

El segundo conglomerado que se puede apreciar en tabla 7 está compuesto por las variables en las cuales se encuentran las fortalezas. Se resalta que las fortalezas residen en la esfera ocupacional, presentando un total del 91,6y de sus ítems cómo fortalezas del programa de bienestar laboral. Es entonces que, podemos inferir que la percepción que tienen los empleados es que la universidad le proporciona las herramientas para el ejercicio de sus funciones, que el programa de bienestar utiliza adecuadamente los diferentes medios disponibles para la divulgación de las comunicaciones y que las actividades programa- das desde bienestar laboral están direccionadas a los empleados.

\section{Conclusiones y Recomendaciones}

El bienestar laboral comprende diversos aspectos de la vida del trabajador, es entonces que existe la necesidad de las empresas en reconocer a los empleados como seres humanos, desde lo que significa "ser humano" y no como "activos" los cuales deben ser eficientemente utilizados para obtener el mejor resultado posible; en esta investigación es de resaltar la problemática encontrada presentada en el bajo impacto personal y familiar que se está encontrando en los empleados objetivo del programa actual de bienestar, acompañado de la poca participación en las actividades ofrecidas por la entidad, y el nulo seguimiento que se le está haciendo a las mismas.

Se resalta que la mayoría de las necesidades de los trabajadores administrativos de la Universidad se encuentran en la esfera socio afectivo y familiar coincidiendo un total del $85,7 \%$ de los ítems evaluados en esta dimensión. Así mismo, las satisfacciones de los trabajadores administrativos respecto al bienestar laboral de la Universidad residen en la esfera ocupacional, presentando un total del 91,6\% de sus ítems cómo fortalezas.

Las estrategias planteadas en este trabajo de investigación tienen como finalidad fortalecer las debilidades encontradas en las diferentes esferas que 
Tabla 7.

Segundo conglomerado - Fortalezas

\begin{tabular}{|c|c|c|c|c|c|c|c|}
\hline No. & ITEMS & $\begin{array}{l}\text { Respuestas } \\
\text { Negativas }\end{array}$ & $\begin{array}{c}\text { Neutralida } \\
\text { d }\end{array}$ & $\begin{array}{c}\text { Respuestas } \\
\text { Positivas }\end{array}$ & $\begin{array}{l}\text { Condición } \\
\text { de } \\
\text { fortaleza }\end{array}$ & $\begin{array}{l}\text { Conglomerad } \\
\text { os }\end{array}$ & $\begin{array}{l}\text { Esfera } \\
\text { inicial }\end{array}$ \\
\hline 4 & $\begin{array}{l}\text { Muestra interés por las } \\
\text { capacitaciones } \\
\text { autocuidado, higiene personal, } \\
\text { autoestima y relaciones } \\
\text { interpersonales programadas } \\
\text { por Bienestar Laboral. }\end{array}$ & 64 & 71 & 105 & SI & 2 & $\begin{array}{c}\text { Esfera socio } \\
\text { afectiva y } \\
\text { familiar }\end{array}$ \\
\hline 14 & $\begin{array}{l}\text { Considera que las actividades } \\
\text { programadas por Bienestar } \\
\text { Laboral fortalecen la relación } \\
\text { trabajo-familia. }\end{array}$ & 50 & 63 & 127 & SI & 2 & $\begin{array}{c}\text { Esfera socio } \\
\text { afectiva y } \\
\text { familiar }\end{array}$ \\
\hline 18 & $\begin{array}{l}\text { Ha participado en algún } \\
\text { proceso de formación o } \\
\text { actualización en competencias } \\
\text { laborales. }\end{array}$ & 78 & 78 & 81 & SI & 2 & $\begin{array}{c}\text { Esfera } \\
\text { personal }\end{array}$ \\
\hline 19 & $\begin{array}{l}\text { Recibe reconocimiento por su } \\
\text { desempeño por parte de la } \\
\text { Universidad. }\end{array}$ & 87 & 48 & 103 & SI & 2 & $\begin{array}{c}\text { Esfera } \\
\text { personal }\end{array}$ \\
\hline 21 & $\begin{array}{l}\text { El programa de Bienestar } \\
\text { Laboral organiza actividades } \\
\text { que desarrollan sus habilidades } \\
\text { y destrezas para un mejor } \\
\text { desempeño profesional y } \\
\text { laboral. }\end{array}$ & 75 & 70 & 95 & SI & 2 & $\begin{array}{c}\text { Esfera } \\
\text { personal }\end{array}$ \\
\hline 22 & $\begin{array}{l}\text { El salario que devenga está } \\
\text { acorde a las funciones que } \\
\text { realiza dentro de la } \\
\text { Universidad. }\end{array}$ & 70 & 59 & 110 & SI & 2 & $\begin{array}{c}\text { Esfera } \\
\text { ocupacional }\end{array}$ \\
\hline 23 & $\begin{array}{l}\text { Considera que la Universidad } \\
\text { le proporciona estabilidad } \\
\text { laboral. }\end{array}$ & 14 & 40 & 186 & SI & 2 & $\begin{array}{c}\text { Esfera } \\
\text { ocupacional }\end{array}$ \\
\hline 24 & $\begin{array}{l}\text { La Universidad le ofrece } \\
\text { óptimas condiciones físicas de } \\
\text { trabajo. }\end{array}$ & 16 & 51 & 172 & SI & 2 & $\begin{array}{c}\text { Esfera } \\
\text { ocupacional }\end{array}$ \\
\hline 25 & $\begin{array}{l}\text { Encuentra en la Universidad un } \\
\text { buen clima laboral. }\end{array}$ & 3 & 50 & 187 & SI & 2 & $\begin{array}{c}\text { Esfera } \\
\text { ocupacional }\end{array}$ \\
\hline
\end{tabular}




\begin{tabular}{|c|c|c|c|c|c|c|c|}
\hline 26 & $\begin{array}{l}\text { Se siente motivado con los } \\
\text { incentivos brindados por la } \\
\text { Universidad. }\end{array}$ & 44 & 70 & 127 & SI & 2 & $\begin{array}{c}\text { Esfera } \\
\text { ocupacional }\end{array}$ \\
\hline 27 & $\begin{array}{l}\text { El estilo de dirección en la } \\
\text { Universidad se caracteriza por } \\
\text { ser de puertas abiertas. }\end{array}$ & 23 & 60 & 157 & SI & 2 & $\begin{array}{c}\text { Esfera } \\
\text { ocupacional }\end{array}$ \\
\hline 28 & $\begin{array}{l}\text { Cuenta con las herramientas } \\
\text { necesarias de seguridad y salud } \\
\text { en el trabajo para el } \\
\text { cumplimiento de sus funciones. }\end{array}$ & 15 & 52 & 172 & SI & 2 & $\begin{array}{c}\text { Esfera } \\
\text { ocupacional }\end{array}$ \\
\hline 29 & $\begin{array}{l}\text { Participa usted en } \\
\text { capacitaciones relacionadas } \\
\text { con el programa de seguridad y } \\
\text { salud en el trabajo. }\end{array}$ & 86 & 44 & 111 & SI & 2 & $\begin{array}{c}\text { Esfera } \\
\text { ocupacional }\end{array}$ \\
\hline 30 & $\begin{array}{l}\text { La Universidad le cumple con } \\
\text { los requisitos legales en } \\
\text { seguridad social (ARL, } \\
\text { SALUD Y PENSION }\end{array}$ & 16 & 24 & 201 & SI & 2 & $\begin{array}{c}\text { Esfera } \\
\text { ocupacional }\end{array}$ \\
\hline 32 & $\begin{array}{l}\text { La Universidad comunica a } \\
\text { través de los diferentes medios, } \\
\text { las actividades programadas } \\
\text { por el área de Bienestar } \\
\text { Laboral. }\end{array}$ & 26 & 77 & 137 & SI & 2 & $\begin{array}{c}\text { Esfera } \\
\text { ocupacional }\end{array}$ \\
\hline 33 & $\begin{array}{l}\text { Las actividades desarrolladas } \\
\text { por el programa de Bienestar } \\
\text { Laboral, llenan sus expectativas } \\
\text { y la de su familia. }\end{array}$ & 41 & 71 & 129 & SI & 2 & $\begin{array}{c}\text { Esfera } \\
\text { ocupacional }\end{array}$ \\
\hline
\end{tabular}

Fuente: elaboración propia.

abarca el departamento de bienestar laboral, apuntando al éxito en la implementación de las mismas, llevando un control de cada una de las actividades de bienestar, evaluando el grado de aceptación y el impacto que tenga en los trabajadores de la Universidad.

A continuación, se describen las estrategias sugeridas resultado del proceso de investigación:

- Presentar horarios flexibles en la programación de actividades de bienestar laboral, para que no exista una barrera entre las labores diarias y la programación de dichas actividades.

- Realizar actividades de Bienestar por sedes específicas, dejando de centralizar las actividades en un solo sitio (sede).
- Creación de un isotipo y de un logotipo que identifique el programa de bienestar laboral, para ser utilizado como herramienta de recordación en las actividades programadas por la oficina de Bienestar.

- Crear hojas de inscripción previas a los futuros participantes que vayan asistir a las actividades y/o eventos.

- Limitar ciertas actividades con una capacidad de cupos disponibles, con una confirmación de la asistencia al evento.

- Utilizar todos los canales de difusión electrónicos (Intranet - Televisores - Fondos de pantallas en los computadores) con que cuen- 
ta la Universidad, para la divulgación de las actividades de Bienestar laboral.

- Utilizar la revista, periódico o carteleras informativas con que cuenta la Universidad en todas las sedes, como medio de divulgación del programa de Bienestar laboral.

- Realizar reuniones programadas por la oficina de Bienestar laboral, con los jefes de las áreas, para comprometer a los líderes de grupos y sus colaboradores a la participación de las actividades propias del programa.

- Realizar seguimientos con los formatos elaborados para tal fin, y llevar estadísticas de las actividades realizadas y el impacto de la misma en la comunidad de empleados.

- Implementación de los formatos de asistencias, evaluación de la actividad y seguimiento de actividades, los cuales están anexados en el cuerpo del trabajo de investigación.

- Publicación en carteleras, redes sociales y medios electrónicos, listado de cumplimentados mensual, adjuntando tarjetas de cumpleaños vía electrónica.

- Implementar indicadores de gestión a las actividades de Bienestar laboral.

- Organizar de la mano del departamento de desarrollo y capacitación, una serie de inducciones y reducción al personal, haciéndoles conocer los beneficios que encuentran en el programa de Bienestar laboral.

\section{Referencias bibliográficas}

Arenas, G. (1993). El bienestar laboral en el marco de la legislación del trabajo y la seguridad social en Colombia. Seminario de bienestar y desarrollo humano en la empresa.

Barrios, S. \& Paravic, T. (2006). Promoción de la salud y un entorno laboral saludable. Revista Latino-Americana de Enfermagem, 14(1), 136-41. https://doi.org/10.1590/S010411692006000100019.

Calderón, G., Murillo, S., \& Torres, K.Y. (2003). Cultura organizacionalybienestarlaboral.CuadernosdeAdministración. Pontificia Universidad Javeriana, 16(25), 109-37. http://www. redalyc.org/articulo.oa?id=20502506.

Campo-Arias, A., \& Oviedo, H. (2008). Propiedades Psicométricas de una Escala: La Consistencia Interna. Revista de Salud Pública, 10(5), 831-39.

Colciencias. (2016). Programa de Bienestar 2016. www.colciencias. gov.co

Danna, K., \& Griffin, R. (1999). Health and Well-Being in the Workplace: A Review and Synthesis of the Literature. Journal of Management, 25(3), 357-84. https://doi. org/10.1177/014920639902500305.

Estrada, Y., \& Miguel, R. (2010). El bienestar laboral y su incidencia en la gestión exitosa de las empresas en el turismo. Revista de Investigación en Turismo y Desarrollo Local, 3(8), 1-12.

Frone, M., Russia, M., \& Cooper, L. (1992). Antecedents and outcomes of work-family conflict: testing a model of the workfamily interface. Journal of Applied Psychology, 77(1), 65-78.

Fundación Española de Ciencia y Tecnología - FECYT. (2002). Manual de Frascati - Propuesta de Norma Práctica Para Encuestas de Investigación y Desarrollo Experimental. OCDE. https://www.ciencia.gob.es/stfls/MICINN/Investigacion/ FICHEROS/ManuaFrascati-2002_sp.pdf

García, M., \& Berrios, P. (1999). El significado del trabajo en personas con patrón de conducta tipo A.” Psychothema, 11(2), 357-66. 
Hernández, R., Fernández, C, \& Baptista, P. (2010). Metodología de la investigación. Quinta ed. México, Ciudad de México: McgrawHill / Interamericana Editores.

Martínez, A. (2008). La influencia de las políticas activas de empleo en las transformaciones de los modos de intervención y los modelos contemporáneos de bienestar social. Portularia: revista de trabajo social, 8(2), 103-15. http://www.uhu.es/ publicaciones/revistas/portularia/.

Mejía Ceballos, J. C., Laca Arocena, F. A., \& Gondra Rezola, J. M. (2009). Factores de personalidad, afectivosy sociodemográficos en la predicción del bienestar laboral de docentes. Psicología y Salud, 19(1), 121-32.

Meliá, J. L. \& Peiró, J. M. (1989). La medida de la satisfacción laboral en contextos organizacionales: el cuestionario de satisfacción S20/23." Psicologemas, 5, 59-74.

Ministerio de Educación Nacional. (2009). Guía para diseño programa de bienestar laboral sector docente. https://www. mineducacion.gov.co/

Orozco, O. A. (2010). Factores psicosociales del ambiente laboral que caracterizan a funcionarios con contratación a término fijo e indefinido de una universidad privada de la ciudad de Cali. Revista Científica Guillermo de Ockham, 8(2), 55-68.

Vigoya, V. (2002). Bienestar Social Laboral. Bogotá, Colombia: Departamento Administrativo de la Función Pública (DAFP). 